\title{
Presurgical Orthopedics in Cleft Lip and Palate Care
}

\author{
LaQuia A. Vinson \\ Department of Pediatric Dentistry, School of Dentistry, Indiana University, United States
}

Copyright $(2016$ by authors, all rights reserved. Authors agree that this article remains permanently open access under the terms of the Creative Commons Attribution License 4.0 international License.

\begin{abstract}
Cleft lip and palate is one of the most common birth defects occurring 1 of every 700 live births. Historically, cleft lip and palate care typically involves the use of presurgical infant orthopedics appliances (PIOA) fabricated by a pediatric dentist as an adjunct to the overall management of the defect approximate the cleft segments prior to surgical repair. However, the necessity of presurgical orthopedics in managing the resulting orofacial deformity is still one that elicits discussion by craniofacial teams due to the variety of methodologies available and the inconsistency in long-term results produced by these devices. Nonetheless, presurgical orthopedics used for patients with cleft lip and palate remains primarily focused on four types' devices which have produced varied results; however, there are other techniques which offer promising results. In spite of contradictory findings, presurgical orthopedics is believed to be a vital influence on growth in cleft life and palate deformities.
\end{abstract}

Keywords Cleft Lip And Palate, Craniofacial Anomalies, Pediatric Dentistry

\section{Introduction}

Craniofacial anomalies are birth defects of the skull and face. Tens of thousands of infants in the United States are affected each year. The most common of these birth defects are cleft lip and/or cleft palate, which occur in approximately one out of every 700 births with 4,437 new cases annually [1,2]. Treatment of cleft lip and/or cleft palate typically requires several interventions throughout the first two decades of life. Although these interventions are necessary for adequate oral and respiratory function, there is a significant cost required for total facial rehabilitation. The overall cost of treatment for a child with a unilateral cleft lip and palate in their first year of life is $\$ 22,744$ [3]. However, with skyrocketing health care costs and continued efforts to increase quality and efficiency in healthcare, the use of presurgical orthopedics in cleft lip and palate care may evolve from an option to a necessity for patients [4].
Presurgical orthopedics can be defined as a type of therapy that "uses forces to reposition tissues secondarily displaced due to a cleft deformity" [5]. Orthopedic appliances were developed to aid in the correction of cleft lip and palate by utilizing both compression and tension forces or passively guiding growth. As stated by Braumann et al, "the aims of presurgical infant orthopedics are to reduce the width of the cleft gap, to achieve an optimal alignment of the cleft segments within the first few months of infancy prior to cheiloplasty, and to allow surgical repair with minimal tension" [6]. The reduction in cleft width can ultimately allow for a surgical result where soft tissues are under less tension and have better surgical repair results $[7,8]$.

Additional benefits of presurgical infant orthopedics include the normalization of feeding and tongue function, better speech development, reduced risk of aspiration, and reduced severity of dental and skeletal deviations [8]. Both Kogo and Turner concluded that infants who received presurgical orthopedic treatment with a maxillary obturator and lactation education as well showed a reduction in feeding time and an increase in fluid volume intact $[9,10]$. Consequently, weight gain is a very important element of an infant's early life, and thus should be reason enough to use at minimum passive presurgical orthopedics. However, concerns regarding the use of presurgical infant orthopedics focus on their cost, long-term effects, restrictions on maxillary growth, negative effects on speech, and benefits not lasting longer than surgical soft palate closure [11-13]. Nonetheless, the debate of using presurgical orthopedics and their positive effects on growth will continue, and the purpose of this article is to review various methods of presurgical orthopedics used in patients with cleft lip and palate.

Presurgical infant orthopedic use was first described by Hoffman in 1686 in which an extraoral anchored headcap was used to place a retraction force on the premaxilla [14]. Contemporary to that was McNeil's used of an intraorally anchored device in 1950 and Berkowitz's description of a head bonnet in 1996 [15]. There has been a continued evolution of presurgical orthopedics since their first introduction into cleft lip and palate care. Over time, a classification system has been developed for the various 
approaches involved in presurgical orthopedic treatment. Infant orthopedics can be categorized as surgical or non-surgical. Presurgical (non-surgical) orthopedic devices can be further classified into two categories: active and passive appliances. Active appliances are fixed orally and apply traction through mechanical methods while passive appliances maintain a distance between the two maxillary segments as an external force is applied [16].

Several authors have reported that presurgical orthopedics have resulted in several benefits to patients with cleft lip and palate such as: a reduction in cleft width by stimulation of patalal shelf growth, improved maxillary arch development, improved growth of the face and infant overall, improved occlusion, feeding, speech, hearing, and language development [17]. Other reasons given for the use of presurgical orthopedics include proper growth and development of the oral cavity, including proper posture for the resting musculature including that of the tongue [18]. Ultimately, the molding that occurs with presurgical orthopedic use can result in a more uniform osseous base [19]. These benefits are believed to facilitate surgical closure and improve the final aesthetic result [20]. However, there are some who believe that active orthopedic treatment in infants with cleft lip and palate will impair facial growth; yet, if maxillary segments are repositioned so that the lip segments are in closer apposition, then the lip surgery should be easier $[21,22]$.

\section{Lip Adhesion Surgery}

Surgical treatment for prexmaxillary retraction began in the 1960s with the advent of lip adhesion surgery [23]. Lip adhesion surgery is considered the most cost effective and simplest presurgical therapy in terms of contouring alveolar segments [24]. Lip adhesion surgery has been another method used in patients born with wide clefts, but it has been criticized for its additional risk with the requirement of an additional surgery and expense to patients: as well as its high rate of dehiscence and scarring: additionally, it is not a uniformly accepted procedure [25]. There is also the possibility that the alveolar segments do not align in an ideal position due to the tension of the surgically adhered lip creating an uncontrolled force: this can ultimately result in a collapse of the dental arches [26].

\section{Latham Appliance}

The Latham appliance, also known as the dentomaxillary advancement (DMA) appliance was developed in 1980. The Latham appliance is an active pin-retained appliance which requires surgical fixation to bone. The Latham appliance was developed to align the alveolar arch through rapid orthopedic correction. The appliance is surgically placed anywhere from 2 to 5 months of age. The Latham appliance works by simultaneously applying pressure to the cleft segments over a 4 to 6 week period to move the alveolar segments into proper position. However, an anterior as well as a posterior cross-bite is an unwarranted side effect that may occur more frequently with Latham appliance usage [27]. Even though long term studies have shown that more anterior and posterior cross-bites occurred in children treated with the Latham appliance, Chan et al concluded that the Latham appliance did not affect dental arch relationships in preadolescent children [28].

\section{Lip Taping}

Lip taping involves using medical adhesive tape such as foam or surgical silk tape or Steri-strips ${ }^{\circledR}$ across the upper lip (Figure 1).

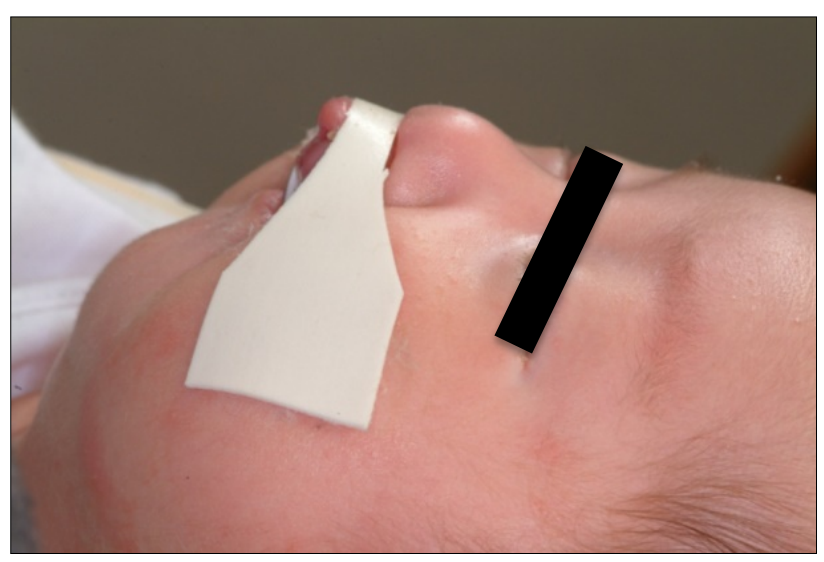

Figure1. Foam tape used for lip taping

Tape is typically placed in the first week of life for patients with exceptionally wide clefts. After the application of a skin adherent or a transparent skin dressing such as Tegaderm ${ }^{\circledR}$, three to four $6-8 \mathrm{~mm}$ wide strips of tape are applied over the area of the cleft lip. The goal of this therapy is to approximate the alveolar segments within 5 $\mathrm{mm}$ of each other [25]. It is believed that lip taping produces a type of "non-surgical lip adhesion" effect [29]. This simple method is believed to be clinically useful, but there are limited controlled studies to support this [5].

\section{Hotz Appliance}

A Hotz appliance also known as the Zurich approach or palatal obturator is a passive orthopedic plate that slowly aligns the cleft segments when used with lip taping.

However, there are studies that have shown that this appliance has failed to prevent collapse of the alveolar segments [5]. The appliance, which is typically fabricated by a pediatric dentist, is made of hard acrylic or a combination of both hard and soft acrylic: it passively covers the alveolar segments and extends slightly into the area of the cleft and the buccal sulci. Children wear the device 24 hours per day, and the obturator is removed only for daily cleaning. Over time, as the infant grows, it is 
possible for the device not to fit as securely as it did when it was initially placed: parents are then advised to use a paste denture adhesive to aid in retention. The Hotz appliance is often used to assist with both bottle-feeding and to allow some breast-feeding in infants with cleft lip and palate. There is no specific research about the success of a Hotz appliance, but it is believed to have a positive impact on cleft patients [11].

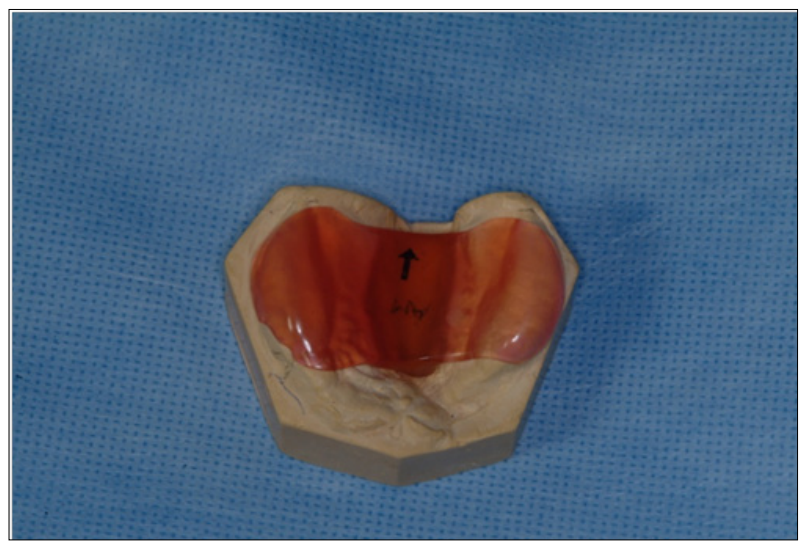

Figure 2. Hotz type appliance on maxillary dental cast

However, there are studies that have shown that this appliance has failed to prevent collapse of the alveolar segments [5]. The appliance, which is typically fabricated by a pediatric dentist, is made of hard acrylic or a combination of both hard and soft acrylic: it passively covers the alveolar segments and extends slightly into the area of the cleft and the buccal sulci. Children wear the device 24 hours per day, and the obturator is removed only for daily cleaning. Over time, as the infant grows, it is possible for the device not to fit as securely as it did when it was initially placed: parents are then advised to use a paste denture adhesive to aid in retention. The Hotz appliance is often used to assist with both bottle-feeding and to allow some breast-feeding in infants with cleft lip and palate. There is no specific research about the success of a Hotz appliance, but it is believed to have a positive impact on cleft patients [11].

\section{NAM (Nasoalveolar Molding)}

Nasoalveolar molding also known as NAM therapy passively repositions both the alveolar segments and nasal cartilages prior to cleft lip repair [30]. NAM therapy, which begins in the first week of life, consists of utilizing a custom acrylic molding plate with retention buttons that is secured to the patient's face using orthodontic elastics and surgical tape. The infant is seen weekly to adjust the molding plate to bring the alveolar segments closer together. Acrylic from the molding plate is removed and soft denture reline material is successively added to approximate the segments over time. When the width of the cleft is reduced to approximately $5 \mathrm{~mm}$, a nasal stent component is added to the device.
The effect of this type of presurgical orthopedics is that a less extensive surgery is required for the lip and nasal repair, and there is less tension on the reconstruction, theoretically resulting in more predictable reconstructive results. Indeed, greater nasal symmetry is be obtained after cleft lip repair using NAM therapy as well as better lip form, reduced oronasal fistulas and labial deformities, and a namely a $60 \%$ reduction in the need for secondary bone grafting [31]. NAM has been demonstrated to be a cost effective means of cleft lip repair when patients are followed long term as well [32]. However, Clark found that while clinical exam showed improved nasal and lip esthetics, three-dimensional stereophotography did not confirm such results [33].

Although NAM therapy is considered to be one of the most effective forms of presurgical orthopedics, it is also one of the most costly and time consuming methods that is only provided by $37 \%$ of craniofacial teams in the United States [16]. NAM is a labor-intensive technique that requires much dedication and extensive involvement of the dental team. NAM therapy requires frequent visits, traditionally weekly, from the time of initial appliance placement until the time of lip repair surgery.

\section{DynaCleft ${ }^{\circledR}$ and Nasal Elevators}

DynaCleft ${ }^{\circledR}$ is a premade nasal and alveolar molding device which can be used to successfully mold the upper lip, alveolus and nose prior to cleft lip repair (Figure 3).

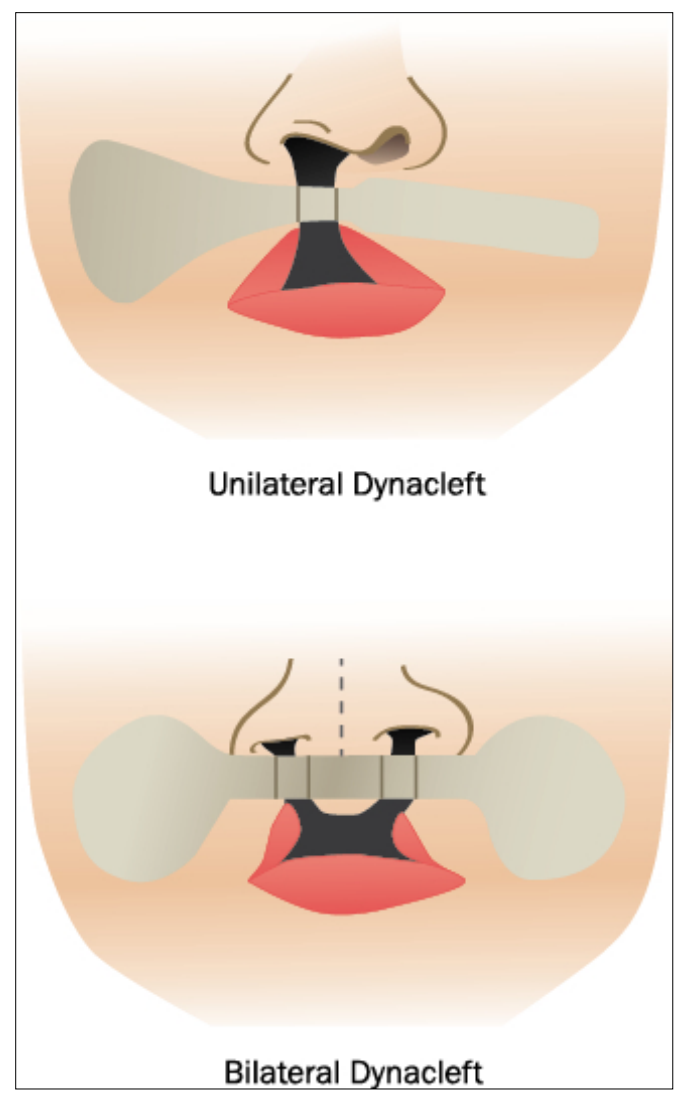

Figure 3. Illustration of unilateral and bilateral DynaCleft ${ }^{\circledR}$ 
While traditional surgical adhesive tape (e.g. Silk tape, Steri-strips $\left.{ }^{\circledR}\right)$ have been used in the past, unlike tape, DynaCleft ${ }^{\circledR}$ offers the benefit of being able to provide a constant approximation force with an elastic center that allows it to conform to a baby's mouth better because of its ability to expand and contract [34]. Additionally, the controlled force that it provides to the prolabium and premaxilla could improve surgical results and decrease the necessity of early lip adhesion surgery. As the DynaCleft ${ }^{\circledR}$ device is premade, there is no need to create custom-made devices for the molding process. Therefore, there is no labor cost associated with DynaCleft ${ }^{\circledR}$ therapy. A recent study by Monasterio et al, DynaCleft ${ }^{\circledR}$ in use with a nasal elevator was able to produce results similar to that of NAM therapy [35]. Nasal elevators were found to improve the shape of the nose and alae as well as reduce the need for primary surgery to the nose in patients with unilateral cleft lip and palate in a study by Abidu et al (Figure 4). Consequently, this technique provides an emerging method of treatment in cleft lip and palate care [36].

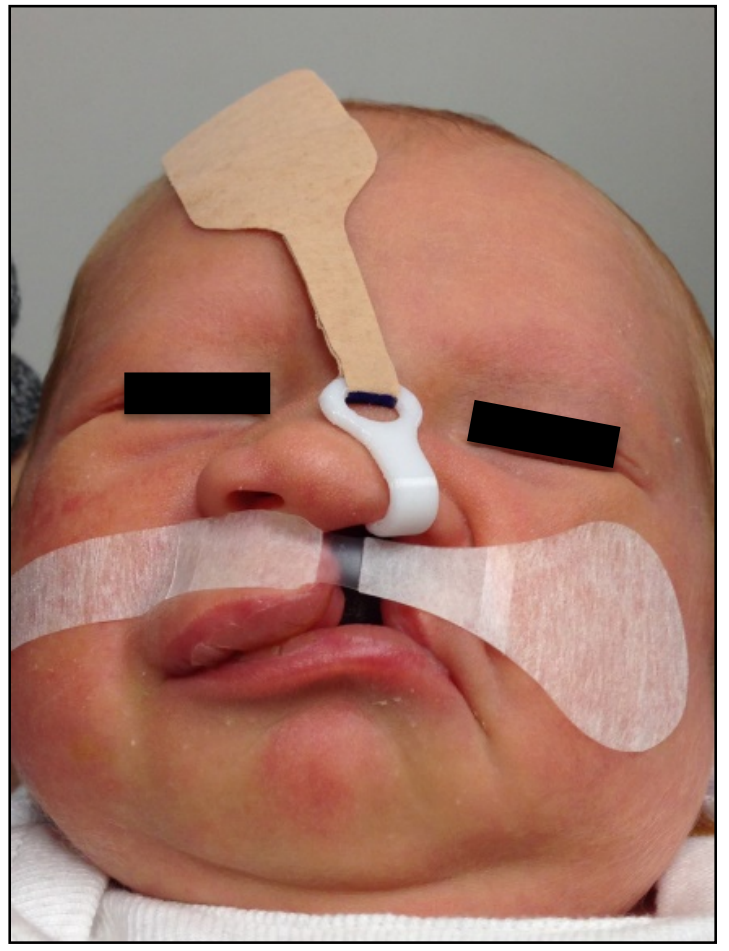

Figure 4. Unilateral DynaCleft ${ }^{\circledR}$ and nasal elevator in place

Even after over 5 decades of treatment and research, there is still no consensus regarding the benefits of presurgical orthopedic therapy in the treatment of patients with cleft lip and palate as well as the long-term effects of treatment [20,37]. Assessing the effect of presurgical orthopedic devices is difficult due to the variety of timing and treatment protocols for surgery and orthodontic treatment [11]. Another reason is that research on presurgical orthopedics is very limited due to small sample sizes as well as many studies have been considered heterogeneous and lacked adequate reporting $[11,17]$.
Although one study demonstrated the alveolar dimensions in children with unilateral cleft lip and palate can approximate those of non-cleft peers with the use of presurgical orthopedics, the Dutchcleft study concluded that there were no differences in the arch forms between the orthopedic and non-orthopedic groups after lip closure $[11,18]$. Despite contradictory findings, it is still widely accepted that presurgical orthopedics is a vital influence on normal physiologic growth in cleft lip and palate deformities, and its use can guide alveolar growth to dimensions that approach those of normal anatomical position and prevent collapse of the alveolar segments. Unfortunately, there exists no agreed upon treatment goals, standards, or protocols [25].

\section{Conclusions}

Even though, the overall benefits of presurgical orthopedics on maxillary growth and development are still debated, pediatric dentists still play a vitally critical role in their use with fabricating them for nearly $35 \%$ of all craniofacial teams in the United States [18, 38-40]. Hopefully, the development of other clinical techniques may make the practice of using presurgical orthopedics in the overall care of children with cleft lip and palate more accessible for craniofacial teams who may have limited resources while achieving the same results of proven methodologies.

\section{REFERENCES}

[1] Johnson CY, Honein MA, Hobbs CA, Rasmussen SA. Prenatal diagnosis of orofacial clefts, National Birth Defects Prevention Study, 1998-2004. Prenat Diagn 29(9):833-9, 2009.

[2] Online available : http://www.webcitation.org/query?url=http $\% 3 \mathrm{~A} \% 2 \mathrm{~F} \% 2 \mathrm{Fwww}$.nidcr.nih.gov\%2FDataStatistics\%2FFin dDataByTopic\%2FCraniofacialBirthDefects\%2FPrevalence Cleft\%2520LipCleftPalate.htm\&date=2016-02-24

[3] Pfeifer TM, Grayson BH, Cutting CB. Nasoalveolar Molding and Gingivoperiosteoplasty versus Alveolar Bone Graft: An Outcome Analysis of Cost in the Treatment of Unilateral Cleft Alveolus" Cleft Palate-Craniofac J 39(1): 26-29, 2002.

[4] Online available : http://www.webcitation.org/query?url=htt p $\% 3 \mathrm{~A} \% 2 \mathrm{~F} \% 2 \mathrm{Fwww}$. hhs.gov\%2Fhealthcare $\% 2$ Ffacts-and-f eatures\%2Fkey-features-of-aca-by-year\%2Findex.html\&date $=2016-02-24$

[5] Jaeger M, Braga-Silva J, Gehlen D, Sato Y, Zuker R, Fisher D. Correction of the Alveolar Gap and Nostril Deformity by Presurgical Passive Orthodontia in the Unilateral Cleft Lip. Ann Plast Surg 59(5): 489-494, 2007.

[6] Braumann B, Keillig L, Bourauel C, Jager A. Three dimensional analysis of morphological changes in the maxilla of patients with cleft lip and palate. Cleft Palate Craniofac J 39:1-11, 2002. 
[7] Millard DR, Latham RA. Improved primary surgical and dental treatment of clefts. Plastic Reconstr Surg. 86:856-871, 1990.

[8] Radhakrishnan V, Sabarinath VP, Thombare P, Hazarey PV, Bonde R, Sheorain A. Presurgical nasoalveolar molding assisted primary reconstruction in complete unilateral cleft lip palate infants. J Clin Pediatr Dent. 34(3):267-74, 2010.

[9] Kogo M, Okada G, Ishii S, Shikata M, Iida S, Matsuya T. Breast-feeding for cleft lip and palate patients using the Hotz-type plate. Cleft Palate Craniofac J. 34:351-353, 1997.

[10] Turner L, Jacobsen C, Humenczuk M, Singhal VK, Moore D, and Bell $\mathrm{H}$. The effects of lactation education and a prosthetic obturator appliance of feeding efficiency in infants with cleft lip and palate. Cleft Palate Craniofac J 38:519-524, 2001.

[11] Esenlik E. Presurgical Infant Orthopedics for Cleft Lip and Palate: A Review. J Surg 11(1)313-18,2015.

[12] Prahl C, Kuijpers-Jagtman AM, van't H MA, Prahl-Andersen B. A randomized prospective clinical trial into the effect of infant orthopaedics on maxillary arch dimensions in unilateral cleft lip and palate. Eur J Ora Sci 109:297-305, 2001.

[13] Hak MS, Sasaguri M, Sulaiman FK, Hardono ET, Suzuki A, Nakamura S, et al. Longitudinal Study of Effect of Hotz's Plate and Lip Adhesion on Maxillary Growth in Bilateral Cleft Lip and Palate Patients. Cleft Palate Craniofac J 49(2):230-6, 2012.

[14] Grayson BG, Maule D. Cleft lip repair: trends and techniques. Semin Plast Surg 19(4):294-301, 2005.

[15] Berkowitz S. A comparison of treatment results in complete bilateral cleft lip and palate using a conservative approach versus Millard-Latham PSOT procedure. Semin Orthod 2(3):169-84, 1996.

[16] Santiago PE, Schuster LA, Levy-Bercowski D. Management of the alveolar cleft. Clin Plast Surg. 41(2):219-32, 2014.

[17] Adali N, Mars M, Noar J, Sommerlad B. Presurgical Orthopedics Has No Effect on Archform in Unilateral Cleft Lip and Palate. Cleft Palate Craniofac J 2012;49(1):5-13.

[18] Kozelj V. The Basis for Presurgical Orthopedic Treatment of Infants with Unilateral Complete Cleft Lip and Palate. Cleft Palate Craniofac J 37(1):26-32, 200.

[19] Marsh JL. Comprehensive Care for Craniofacial Anomalies. Chicago: Yearbook Medical Publishers; 1980:13.

[20] Uzel A, Alparsian ZN. Long-Term Effects of Presurgical Infant Orthopedics in Patients with Cleft Lip and Palate: A Systematic Review. Cleft Palate Craniofac J 48(5):587-595, 2011.

[21] Chan KT, Hayes C, Shusterman S, Mulliken JB, Will LA. The effects of active infant orthopedics on occlusal relationships in unilateral complete cleft lip and palate.Cleft Palate Craniofac J. 40(5):511-7, 2003.

[22] Ross RB, MacNamera MC. Effect of presurgical infant orthopedics on facial esthetics in complete bilateral cleft lip and palate. Cleft Palate Craniofac J 31:68-73, 1994.

[23] CH Thorne, RW Beasley, SJ Aston, SP Bartlett, GC Geoffrey, SL Scott. Grabb and Smith's Plastic Surgery, 6th Edition, Lippincott Williams \& Wilkins, USA, 2007.

[24] Gatti GL, Lazzeri D, Romeo G, Balmelli B, Massei A. Effect of lip adhesion on maxillary arch alignment and reduction of a cleft's width before definitive cheilognathoplasty in unilateral and bilateral complete cleft lip. Scand J Plast Reconstr Surg Hand Surg. 44(2):88-95, 2010.

[25] Pool R, Farnworth TK. Preoperative Lip Taping the Cleft Lip. Ann Plast Surg 32-: 243-249, 1994.

[26] Pruzansky S. Presurgical orthopedics and bone grafting for infants with cleft lip and palate: A dissent. Cleft Palate Craniofac J 1:164-187, 1964.

[27] Berkowitz S, Mejia M, Bystrik A. A comparison of the effects of the Latham-Millard procedure with those of a conservative treatment approach for dental occlusion and facial aesthetics in unilateral and bilateral complete cleft lip and palate: part I. Dental occlusion. Plast Reconstr Surg 113:1-18, 2004.

[28] Chan KT, Hayes C, Shusterman S, Mulliken JB, Will LA. The effects of active infant orthopedics on occlusal relationships in unilateral complete cleft lip and palate. Cleft Palate Craniofac J. 40(5):511-7, 2003.

[29] Aminpour S, Tollefson TT. Recent advances in presurgical molding in cleft lip and palate. Curr Opin Otolaryngol Head Neck Surg 16:339-346, 2008.

[30] Grayson BH, Santiago PE, Brecht LE, Cutting CB. Presurgical nasoalveolar molding in infants with cleft lip and palate. Cleft Palate Craniofac J 36(6):486-98, 1996.

[31] Barillas I, Dec W, Warren SM, Cutting CB, Grayson BH. Nasoalveolar molding improves long-term nasal symmetry in complete unilateral cleft lip-cleft palate patients. Plast Reconstr Surg. 123(3):1002-6, 2009.

[32] Pfeifer TM, Grayson BH, Cutting CB. Nasoalveolar molding and gingivoperiosteoplasty versus alveolar bone graft: an outcome analysis of costs in the treatment of unilateral cleft alveolus. Cleft Palate Craniofac J. 39(1):26-9, 2002.

[33] Clark SL, Teichgraeber JF, Fleshman RG, et al. Long-term treatment outcome of presurgical nasoalveolar molding in patients with unilateral cleft lip and palate. J Craniofac Surg. 22(1):333-6, 2011

[34] Online available : http://www.webcitation.org/query?url=http $\% 3 \mathrm{~A} \% 2 \mathrm{~F} \% 2 \mathrm{Fsou}$ thmedic.com $\% 2$ Fproducts $\% 2$ Fdynacleft-cleft-lip-approximat ion $\% 2 F \&$ date $=2015-11-06$

[35] Monasterio L, Ford A, Gutiérrez C, Tastets ME, García J. Comparative Study of Nasoalveolar Molding Methods: Nasal Elevator Plus DynaCleft Versus NAM-Grayson in Patients With Complete Unilateral Cleft Lip-Palate. Cleft Palate Craniofac J. 50(5):548-54, 2013.

[36] Abdiu A, Ohannessian P, Berggren A. The nasal alar elevator: A new device that may reduce the need for primary operation of the nose in patients with cleft lip. Scand J Plast Reconstr Surg Hand Surg, 43:71-74, 2009.

[37] Albino FP, Koltz PF, Girotto JA. Predicting Out-of-Pocket Costs in the Surgical Management of Orofacial Clefts. Plast Reconstr Surg 126(4):188e-189e, 2010.

[38] Mishima K, Sugahara T, Mori Y, Sakuda M. Three-dimensional comparison between the palatal forms in complete unilateral cleft lip and palate with and without Hotz plate from cheiloplasty to palatoplasty. Cleft Palate Craniofac J. 33:312-317, 1996. 
[39] Mishima K, Mori Y, Sugahara T, Sakuda M. Comparison between the palatal configurations in complete and incomplete unilateral cleft lip and palate infants under 18 months of age. Cleft Palate Craniofac J. 38:49-54, 2001.
[40] Raju R, Tate AR. The Role of Pediatric Dentistry in Multidisciplinary Cleft Palate Teams at Advanced Pediatric Dental Residency Programs. Pediatr Dent 31:188-92, 2009 